\title{
An Upper Bound on the Average Size of Silhouettes
}

\author{
M. Glisse · S. Lazard
}

Received: 21 December 2006 / Revised: 26 April 2008 / Accepted: 2 May 2008 /

Published online: 20 June 2008

(C) Springer Science+Business Media, LLC 2008

\begin{abstract}
It is a widely observed phenomenon in computer graphics that the size of the silhouette of a polyhedron is much smaller than the size of the whole polyhedron. This paper provides, for the first time, theoretical evidence supporting this for a large class of objects, namely for polyhedra or, more generally, tessellated surfaces that approximate surfaces in some reasonable way. The approximated surfaces are twomanifolds that may be nonconvex and nondifferentiable and may have boundaries. The tessellated surfaces should, roughly speaking, have no short edges, have fat faces, and the distance between the mesh and the surface it approximates should never be too large. We prove that such tessellated surfaces of complexity $n$ have silhouettes of expected size $O(\sqrt{n})$, where the average is taken over all points of view. The viewpoints can be chosen at random at infinity or at random in a bounded region.
\end{abstract}

Keywords Silhouette - Apparent boundary · Rim · Profile · Contour generator · Polyhedron · Upper bound · Average

\section{Introduction}

The silhouette of a polyhedron with respect to a given viewpoint is, roughly speaking, the set of edges incident to a front face and a back face. Silhouettes arise in various problems in computer graphics such as hidden surface removal and shadow computations (see [4-6] for some recent references), and algorithms to compute them

\footnotetext{
M. Glisse · S. Lazard (凶)

INRIA Nancy Grand Est, Université Nancy 2, LORIA, Campus Scientifique, B.P. 239, 54506 Nancy, France

e-mail: Sylvain.Lazard@1oria.fr

M. Glisse

e-mail: Marc.Glisse@loria.fr
} 

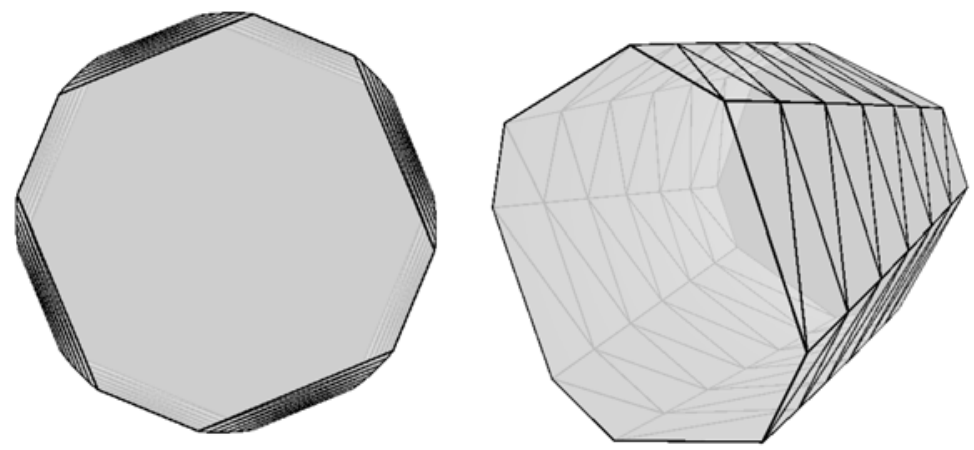

Fig. 1 A worst-case linear silhouette (left) of a polyhedron approximating a cylinder

efficiently have been well studied (see the survey by Isenberg et al. [7]). They are important in shape recognition; Sander et al. [11] claim that the silhouette "is one of the strongest visual cues of the shape of an object."

It is a widely accepted fact that the silhouette of a polyhedron is usually much smaller than the whole polyhedron. Sander et al. [11], for instance, state the largely repeated claim that the silhouette of a mesh is often of size $\Theta(\sqrt{n})$, where $n$ is the number of faces of the mesh. An experimental study by Kettner and Welzl [9] confirms this for a set of realistic objects. This experimental study was extended by McGuire [10] to a larger database of larger objects for which the observed size of the silhouette is approximately $n^{0.8}$.

There are few theoretical results supporting these observations. Kettner and Welzl [9] prove that a convex polyhedron that approximates a sphere with Hausdorff distance $\varepsilon$ has $\Theta(1 / \varepsilon)$ edges, and a random orthographic projection of such a polytope has $\Theta(1 / \sqrt{\varepsilon})$ silhouette edges. Alt et al. [1] give conditions under which it can be proved that the average silhouette of a convex polyhedron has size $O(\sqrt{n})$ and give additional conditions under which the worst-case size is provably sub-linear.

The goal of this paper is to study the average silhouette size of nonconvex polyhedra. Convexity is a very strong assumption, which was crucial in the previous theoretical results. Here, rather, we assume that the polyhedron is a good approximation of some fixed (not necessarily convex) surface. Notice that it is very difficult to guarantee anything on the worst-case complexity of the silhouette of a polyhedron unless it approximates a strictly convex surface. Alt et al. [1] give an example of a polyhedral approximation of a section of a cylinder with worst-case silhouette size $\Theta(n)$ (see Fig. 1). Moreover, their example can be modified in such a way that the surface is smooth, and its polyhedral approximation is as "nice" as one might hope (for instance, it can be required that the faces are fat triangles that all have almost the same size).

In this paper, we prove an upper bound on the expected size of the silhouette for random viewpoints. We prove that the silhouette of a polyhedron that approximates a surface in a reasonable way has expected size $O(\sqrt{n})$. The average is taken over all viewpoints for a given surface and not on a set of surfaces. The views can be orthographic (i.e., the viewpoints are chosen at random at infinity) or projective (i.e., the viewpoints are chosen at random in a bounded region). The approximated surface is 
not necessarily everywhere differentiable and may have boundaries. The polyhedron (or tessellated surface if the surface has boundaries) should, roughly speaking, have no short edges, its faces should be fat, and the distance between the mesh and the surface it approximates should never be too large.

In Sect. 2, we define precisely the notion of silhouette for polyhedra and general surfaces. We then present and prove our main result in Sect. 3 and conclude in Sect. 4.

\section{Definitions}

The term silhouette has been used in the literature to represent several different notions, depending on the application, reflecting such issues as: is the object considered opaque or transparent? Is occlusion taken into account? Is one interested by what the eye perceives, i.e., a plane curve, or by the space curve which gave birth to it? In the area of photography, for instance, a silhouette (also called apparent boundary) is defined as an outline of a solid object, as cast by its shadow, that appears dark against a light background (Fig. 2(a)). In the field of computer vision, by contrast, the silhouette (also called rim, profile, or contour generator) is roughly defined as the curve on the surface that separates front face regions from the back ones, either for opaque (Fig. 2(b)) or for transparent (Fig. 2(c)) objects.

In this paper, we prove an upper bound on the size of the transparent silhouette; since such a silhouette contains the apparent boundary and the contour, our bounds also apply to all these types of silhouettes. In the rest of the paper, the term silhouette will be used to mean transparent silhouette.

In the rest of this section, we give a formal definition of silhouettes of polyhedra and then provide a definition for more general surfaces.

\subsection{Polyhedra}

The (transparent) silhouette of a polyhedron from a viewpoint (possibly at infinity) is the set of edges that are adjacent to a front face and a back face. A face is considered a front face if the angle between its normal vector and a vector from a point of the face

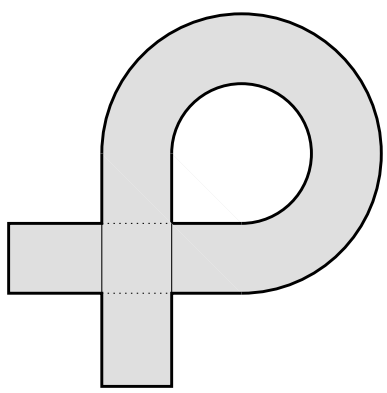

(a)

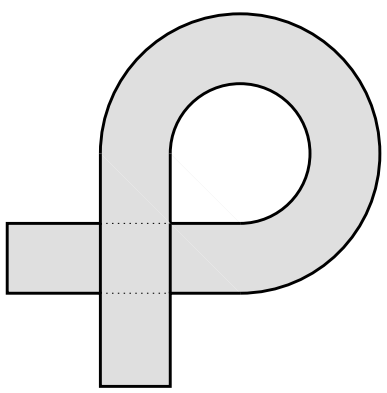

(b)

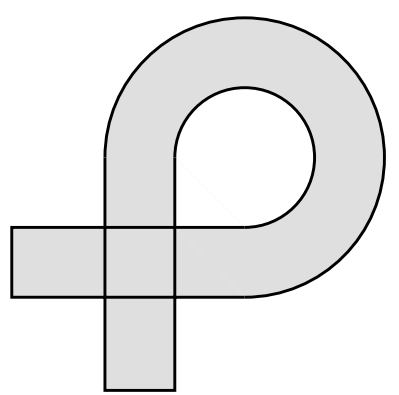

(c)

Fig. 2 Three different notions of silhouettes: (a) outline of a solid, as cast by its shadow, (b) rim of an opaque object, and (c) rim of a transparent object 
to the viewpoint is acute, and a back face if that angle is larger than $\pi / 2$. If the point of view is in the plane containing the face, we refer to the definition of silhouettes for the case of general surfaces. The normal vectors should point outwards, but what really matters is that the orientation is consistent for the two faces that share this edge, so this definition also applies to nonorientable (necessarily self-intersecting) polyhedra.

In this paper, we call the complexity of a silhouette (of a polyhedron) its number of edges.

\subsection{General Surfaces}

Our objective is to bound the size of the silhouette of a polyhedron. To achieve this goal, we need to relate the silhouette of the polyhedron to the silhouette of the surface it approximates, which means we need a definition of silhouettes that applies to a larger class of objects. Although this may seem unintuitive, we first define the silhouette as a set of rays and then relate this to the more usual concept of a set of points on the surface.

Let $S$ be a compact 2-manifold without boundary. It separates $\mathbb{R}^{3}$ in two nonempty open regions; call $\mathcal{O}$ and $\mathcal{O}^{\prime}$ their closures (so $\mathcal{O} \cap \mathcal{O}^{\prime}=S$ and $\mathcal{O} \cup \mathcal{O}^{\prime}=\mathbb{R}^{3}$ ). Let $V$ be a viewpoint not on $S$ but possibly at infinity. The (transparent) silhouette of $S$ from $V$ is the set of rays $R$ starting from $V$ that are tangent to $S$ in a noncrossing way ( $R$ may cross $S$ elsewhere). More formally, we require that there exists an open segment $u$ of $R$ that contains a connected component of $R \cap S$ and is contained either in $\mathcal{O}$ or $\mathcal{O}^{\prime}$.

This definition defines a set of rays. The silhouette can also be seen as the trace of this set of rays on the surface. More precisely, for each ray $R$ on the silhouette, we consider the closest point to $V$ on each connected component of $R \cap S$ that satisfies the noncrossing property. This definition is consistent with the one given for the particular case of polyhedra and is the one we will use in this paper.

For a given viewpoint at infinity, we define the (projected) length of the silhouette as the length (counted with multiplicity if several points have the same projection) of the projection of the silhouette, along the direction given by the viewpoint, on an orthogonal plane.

Remark The definition of the silhouette can be extended to cases where $S$ is not a 2-manifold but an immersion of a compact 2-manifold. More precisely, we have a 2-manifold $S^{\prime}$ and a map $f: S^{\prime} \rightarrow \mathbb{R}^{3}$ such that $S=f\left(S^{\prime}\right)$ and, for any point on $S^{\prime}$, there exists a neighborhood $U$ of that point such that $U$ and $f(U)$ are homeomorphic. The local orientation is sufficient to decide whether $R$ crosses $S$ or not (note that more complicated things can happen than crossing or being tangent, even with smooth surfaces; for instance, the surface may ripple an infinite number of times in the neighborhood of a point, making it impossible to define on which side of $S R$ is near the intersection point). This remark extends to the whole paper and, in particular, to Theorem 1. However, we do not give either a definition or a proof of this, as it would uselessly make everything more obscure. 


\section{Main Results}

Let $S$ be a compact 2-manifold without boundary whose silhouettes have finite average length, $\operatorname{silh}(S)$, where the average is taken over all viewpoints at infinity. Let $P_{n}$ be a polyhedron with $n$ triangular faces, which is homeomorphic to $S$ through $f_{n}: P_{n} \rightarrow S$ and such that:

1. The length of any edge of $P_{n}$ is at least $\frac{\alpha}{\sqrt{n}}$,

2. The faces of $P_{n}$ are fat, and

3. For any $x$ on $P_{n}, d\left(x, f_{n}(x)\right)<\frac{\gamma}{n}$,

where $\alpha$ and $\gamma$ are two arbitrary positive numbers, and $d()$ denotes the Euclidean distance. We actually consider, in the following, polyhedra $P_{n}$ that satisfy Hypothesis 1 and, instead of Hypotheses 2 and 3, the following weaker, ${ }^{1}$ though less intuitive, hypothesis:

4. For any point $x$ on $P_{n}, d\left(x, f_{n}(x)\right)<\frac{\beta h(x)}{\sqrt{n}}$, where $h(x)$ is the smallest height of the triangle(s) of $P_{n}$ that contain(s) $x$, and $\beta$ is an arbitrary positive number.

Theorem 1 The expected complexity of the silhouette of $P_{n}$ is $O(\sqrt{n})$, where the average is taken over all viewpoints at infinity. More precisely, for any $n$, the expected complexity is at most

$$
\left(15 \beta+\frac{24}{\alpha} \operatorname{silh}(S)\right) \sqrt{n} .
$$

Note that the bound is valid for any $n$ and any polyhedron $P_{n}$ satisfying the above assumptions. Note also that the bound depends on $S$ only by the average length of its silhouette.

We first discuss, in Sect. 3.1, the meaning of the hypotheses on $P_{n}$ and their implications. We then prove Theorem 1 in Sect. 3.2. We finally show in Sect. 3.3 how Theorem 1 can be generalized to surfaces with boundary and viewpoints at finite distance. In particular, we prove the following result.

Let $S^{\prime}$ be any compact two-manifold with boundary of finite length and whose silhouette has finite average length (taken over all viewpoints at infinity).

Theorem 2 Any mesh $P_{n}$ with $n$ triangular faces that approximates $S^{\prime}$ according to Hypotheses 1 and 4 has a silhouette of expected complexity $O(\sqrt{n})$ when the viewpoint is chosen uniformly at random in a bounded region.

\subsection{Meaning of the Hypotheses}

Hypothesis 1 is here to avoid short edges. The main idea of the proof is to link the complexity of the silhouette to its length, and arbitrarily short edges would make this impossible. Now the $\frac{1}{\sqrt{n}}$ factor makes sense: intuitively, since the polyhedron has

\footnotetext{
${ }^{1}$ Indeed, for any $x$ in $P_{n}$, Hypotheses 1 and 2 imply that $h(x) \geq \delta / \sqrt{n}$ for some positive constant $\delta$; Hypothesis 4 then follows from Hypothesis 3 , since $h(x) / \sqrt{n} \geq \delta / n>\delta / \gamma \cdot d\left(x, f_{n}(x)\right)$.
} 


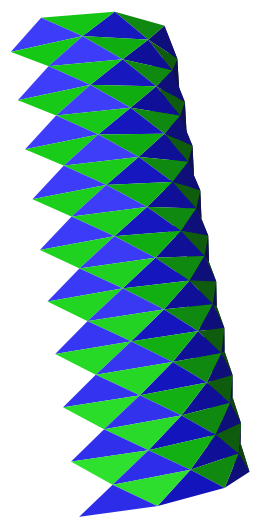

(a)

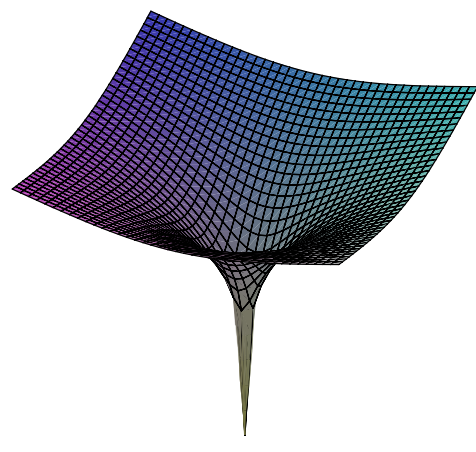

(b)

Fig. 3 (a) Two half lanterns of Schwarz (courtesy of Boris Thibert). (b) A surface that cannot be approximated with the right properties

$n$ faces, each face has area of order $\frac{1}{n}$, which means that the edges have length of $\operatorname{order} \frac{1}{\sqrt{n}}$.

Hypothesis 4 is rather technical, and we discuss instead the meaning of the more intuitive Hypotheses 2 and 3. Hypothesis 2 is quite natural. Hypothesis 3 ensures that $P_{n}$ approximates $S$. Furthermore, the $\frac{1}{n}$ factor is reasonable; indeed, in $2 \mathrm{D}$, when considering a regular polygon with edge length $\Theta\left(\frac{1}{\sqrt{n}}\right)$ inscribed in a circle of radius 1 , the maximal distance between a point on the polygon and the circle is $\Theta\left(\frac{1}{n}\right)$. The situation is the same in 3D. Basically this means that the error when approximating the surface with a plane is of the second order.

Our hypotheses (1-2-3 or 1-4) ensure that the homeomorphism $f_{n}$ has good properties, that is, that, roughly speaking, the polyhedron can be obtained by only a small perturbation of the surface while keeping the normal vectors in approximately the same directions. This is crucial for our proof, since otherwise, for example, a cylinder can be approximated by a lantern of Schwarz [12] (see Fig. 3(a)), where the triangles are not fat, and whose silhouette has expected complexity $\Theta(n)$ and unbounded length.

Notice that the existence of polyhedra with arbitrarily large number of edges that approximate the surface according to these hypotheses is a constraint on the surface. Not every surface admits such an approximation (think of the neighborhood of 0 in the surface defined by $z=\left(x^{2}+y^{2}\right)^{1 / 8}$, which cannot be well approximated with fat triangles as shown in Fig. 3(b)). However, the class of surfaces for which such approximations exist is quite large. It includes, in particular, smooth surfaces and polyhedra with fat faces. 


\subsection{Proof of Theorem 1}

We consider a point of view chosen randomly at infinity. We call $l_{e}$ the length of an edge $e$ of polyhedron $P_{n}$ and $\theta_{e}$ the exterior dihedral angle associated to $e$ (see Fig. 4).

Let $T_{e}$ denote the union of the two triangles adjacent to edge $e$ (including $e$ but not the other edges). For any part $\mathcal{R}$ of $S$, let $\operatorname{silh}(\mathcal{R})$ be the average length of the part of the silhouette of $S$ that lies in $\mathcal{R}$.

We first recall a classical formula on the expected size of silhouettes, which can also be found, for instance, in [10].

An edge $e$ is on the silhouette if the direction of view is in the dark area of the sphere of directions of Fig. 4(b). The angular measure of this region is $4 \theta_{e}$, which means that the probability for $e$ to be on the silhouette is $\theta_{e} / \pi$. The expected number of edges on the silhouette is thus

$$
E=\frac{1}{\pi} \sum_{\text {edge } e} \theta_{e}
$$

We now state our main lemma. The general idea of this lemma is that, under strong hypotheses ( $S$ has bounded curvature, the edges have length $\Theta\left(\frac{1}{\sqrt{n}}\right)$, and Hypotheses 2 and 3 are satisfied), one can prove that $\theta_{e} \leq \frac{C}{\sqrt{n}}$ for some constant $C$. In cases where this inequality does not hold, edge $e$ is near some kind of edge of the surface, or at least some feature that will appear quite often on the silhouette, and we are going to charge this edge to the silhouette of $S$.

Lemma 3 For any edge $e$ on $P_{n}$,

$$
\theta_{e} \leq \frac{C}{\sqrt{n}}+\frac{8 \pi}{l_{e}} \operatorname{silh}\left(f_{n}\left(T_{e}\right)\right) \quad \text { with } C=31.3 \beta
$$

Theorem 1 follows from Lemma 3. Indeed, since $P_{n}$ has $\frac{3 n}{2}$ edges, each of length at least $\frac{\alpha}{\sqrt{n}}$ (by Hypothesis 1), we get by (1) that the expected complexity of the

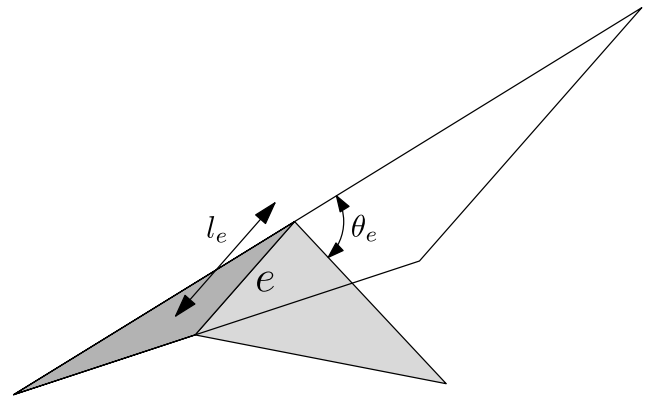

(a)

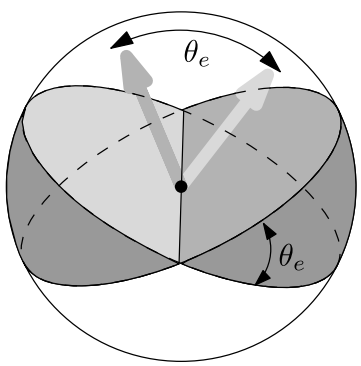

(b)

Fig. 4 (a) Length and dihedral angle of an edge; (b) set of directions for which $e$ is on the silhouette 


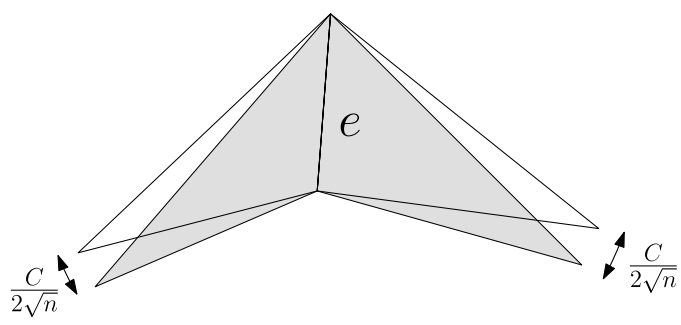

(a)

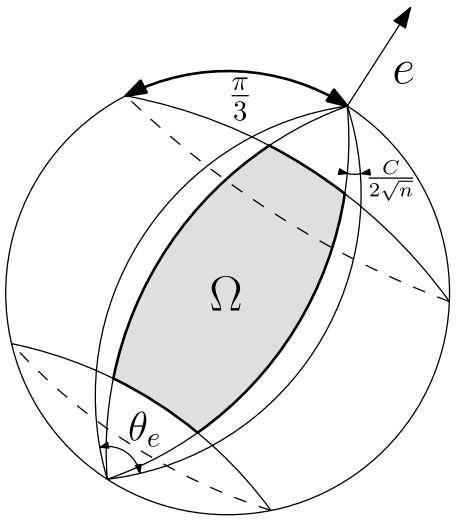

(b)

Fig. 5 Construction of $\Omega$

silhouette is

$$
E \leq \frac{1}{\pi} \frac{3 n}{2} \frac{C}{\sqrt{n}}+8 \frac{\sqrt{n}}{\alpha} 3 \operatorname{silh}(S),
$$

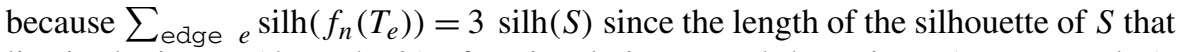
lies in the image (through $f_{n}$ ) of a triangle is counted three times (once per edge). Hence,

$$
E \leq\left(15 \beta+\frac{24}{\alpha} \operatorname{silh}(S)\right) \sqrt{n}=O(\sqrt{n})
$$

Proof of Lemma 3 The idea of the proof is as follows. Consider the set of directions for which $e$ is on the silhouette. We first construct a subset $\Omega$ of these directions whose measure is a constant times $\theta_{e}-\frac{C}{\sqrt{n}}$ (see Fig. 5). We then prove a lower bound on the length of the silhouette of $f_{n}\left(T_{e}\right)$ for all these directions and deduce the result.

Let $C$ be a positive constant, whose value will be defined later (see (3) at the end of Sect. 3.2). For any edge $e$ on $P_{n}$, we can assume that $\theta_{e}-\frac{C}{\sqrt{n}}>0$, since, otherwise, $\theta_{e} \leq \frac{C}{\sqrt{n}}$, and there is nothing else to prove.

The set of directions for which $e$ is on the silhouette is the set of directions between the planes defined by the faces adjacent to $e$. Rotate each face about $e$ by an angle of $\frac{C}{2 \sqrt{n}}$ so that the exterior dihedral angle decreases by $\frac{C}{\sqrt{n}}$ (see Fig. $\left.5(\mathrm{a})\right) . \Omega$ is defined to be the set of directions between these two new planes that make an angle larger than $\pi / 3$ with the line supporting $e$; Fig. 5(b) shows one component of $\Omega$, the other one consists of the symmetric set of opposite directions. The measure of the set of directions between these two planes is $4\left(\theta_{e}-\frac{C}{\sqrt{n}}\right)$. Restricting this set of directions to those that make an angle larger than $\pi / 3$ with the line supporting $e$, we get, by integrating on the sphere of directions, that the measure of $\Omega$ is $2\left(\theta_{e}-\frac{C}{\sqrt{n}}\right)$.

The remaining step uses the property, which we prove in Corollary 5 , that for all the directions in $\Omega$, the silhouette of $f_{n}\left(T_{e}\right)$ has length at least $l_{e} / 4$. Assuming this 


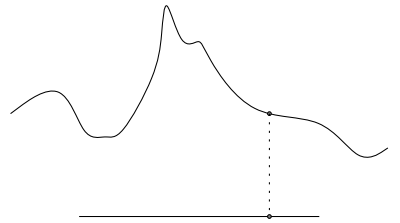

(a) Orthogonal projection along $d$ of $e^{\prime}$ and of the silhouette of $f_{n}\left(T_{e}\right)$.

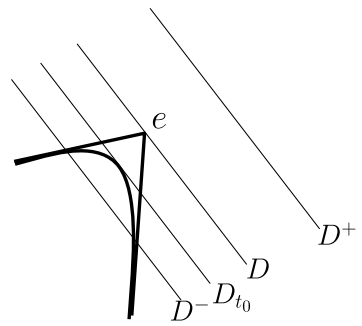

(b) For the definition of $D_{t}$.

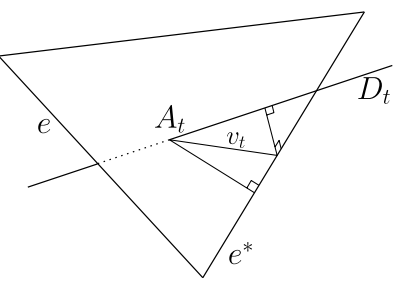

(c)

Fig. 6 For the proofs of Lemma 4 and Corollary 5

temporarily, we integrate this inequality over $\Omega$. The smaller side of the inequality is $2 \frac{l_{e}}{4}\left(\theta_{e}-\frac{C}{\sqrt{n}}\right)$. The larger side is the integral of the length of the silhouette of $f_{n}\left(T_{e}\right)$ over all directions in $\Omega$, which is smaller than this same integral over all directions, that is, $4 \pi \operatorname{silh}\left(f_{n}\left(T_{e}\right)\right)$. Hence, $4 \pi \operatorname{silh}\left(f_{n}\left(T_{e}\right)\right) \geq \frac{l_{e}}{2}\left(\theta_{e}-\frac{C}{\sqrt{n}}\right)$, which concludes the proof.

We now state a lemma and its corollary which we used in the proof of Lemma 3 under the hypothesis that $\theta_{e}-\frac{C}{\sqrt{n}}>0$. We can thus assume in the sequel that this property holds.

Let $e^{\prime}$ be the segment obtained by clipping from $e$ all the points at distance less than $\frac{l_{e}}{4}$ from its endpoints. Refer now to Figs. 6(a)-(b).

Lemma 4 Any line with direction $d \in \Omega$ that intersects $e^{\prime}$ can be translated in a direction orthogonal to $e$ and $d$ until it becomes tangent to $S$ in $f_{n}\left(T_{e}\right)$.

Corollary 5 For any direction $d$ in $\Omega$, the silhouette of $f_{n}\left(T_{e}\right)$ has length at least $\frac{l_{e}}{4}$.

Proof Consider the projection of $e^{\prime}$ and of the silhouette of $f_{n}\left(T_{e}\right)$ onto a plane orthogonal to $d$ (see Fig. 6(a)). It follows from Lemma 4 that, in that plane, each point on the projection of $e^{\prime}$ maps to a point on the projected silhouette in the direction orthogonal to $e^{\prime}$. Hence, the projected silhouette is longer than the projection of $e^{\prime}$, which is at least $\frac{\sqrt{3}}{2}$ times the length of $e^{\prime}$, since $d$ makes an angle of at least $\pi / 3$ with $e^{\prime}$. Thus the silhouette of $f_{n}\left(T_{e}\right)$ has length at least $\frac{\sqrt{3}}{2} \frac{l_{e}}{2}>\frac{l_{e}}{4}$.

Proof of Lemma 4 Let $D$ denote a line with direction $d \in \Omega$ that intersects $e^{\prime}$. Let $T_{1}$ and $T_{2}$ denote the two triangles adjacent to $e$, and let $h_{1}$ and $h_{2}$ denote their respective smallest heights. Let $\chi_{i}=\beta h_{i} / \sqrt{n}, \chi^{+}=\max \left(\chi_{1}, \chi_{2}\right)$, and $\chi^{-}=\min \left(\chi_{1}, \chi_{2}\right)$. Refer now to Fig. 6(b). We call $D_{t}, t \in\left[-\chi^{-}, \chi^{+}\right]$, the line obtained by translating $D$ at distance $|t|$ in a direction orthogonal to the plane defined by $e$ and $d$; positive values of $t$ correspond to lines in the half-space bounded by the plane defined by $e$ and $D$, and not containing $T_{e}$; negative values of $t$ correspond to lines in the other half-space. For clarity, we denote $D_{-\chi^{-}}$by $D^{-}$and $D_{\chi^{+}}$by $D^{+}$. 
Note first that $D^{+}$does not intersect $f_{n}\left(T_{e}\right)$, since $D^{+}$is at distance $\chi^{+}$from $T_{e}$ (by construction) and $d\left(x, f_{n}(x)\right)<\chi^{+}$for any $x$ in $T_{e}$ (by Hypothesis 4 ). We prove that $D^{-}$intersects $f_{n}\left(T_{e}\right)$ and that no line $D_{t}$ intersects the boundary of $f_{n}\left(T_{e}\right)$. This will imply that, sweeping $D_{t}$ from $D^{+}$to $D^{-}$, the first line $D_{t_{0}}$ that intersects $f_{n}\left(T_{e}\right)$ is tangent to $f_{n}\left(T_{e}\right)$ at one of its interior point, which will conclude the proof.

We first prove that no line $D_{t}$ intersects the boundary of $f_{n}\left(T_{e}\right)$. In other words, we prove that, for each edge $e^{*}$ on the boundary of $T_{e}$, no line $D_{t}$ intersects $f_{n}\left(e^{*}\right)$. Let $T_{i}$ be the triangle (of $T_{e}$ ) containing $e^{*}$. By Hypothesis 4 , it is sufficient to prove that the distance between $D_{t}$ and $e^{*}$ remains greater than or equal to $\chi_{i}$ for all $t$.

First notice that it is sufficient to prove that the distance between $D_{t}$ and $e^{*}$ remains greater than or equal to $\chi_{i}$ for all $t \in\left[-\chi^{-}, 0\right]$. Indeed, then, the distance between $D_{0}=D$ and $e^{*}$ is at least $\chi_{i}$, and the distance between $D_{t}$ and $e^{*}$ increases for $t \geq 0$ (see Fig. 6(b)).

Let $\Gamma$ be the smallest angle $d$ can make with the plane containing $T_{i}$ and refer to Fig. 6(b). Let $A_{t}$ be the point of intersection between $D_{t}$ and the plane containing $T_{i}$ and $v_{t}$ be the distance between $A_{t}$ and the point on $e^{*}$ that realizes the distance between $D_{t}$ and $e^{*}$. The distance between $D_{t}$ and $e^{*}$ satisfies $d\left(D_{t}, e^{*}\right) \geq v_{t} \sin \Gamma \geq$ $d\left(A_{t}, e^{*}\right) \sin \Gamma$. Hence, for proving that $d\left(D_{t}, e^{*}\right) \geq \chi_{i}$ for $t \leq 0$, it is sufficient to prove that $d\left(A_{t}, e^{*}\right) \geq \frac{\chi_{i}}{\sin \Gamma}$ for $t \leq 0$. We set $a=\frac{\chi_{i}}{\sin \Gamma}$ to simplify the notation.

We just proved that $d\left(A_{t}, e^{*}\right) \geq a$ implies $d\left(D_{t}, e^{*}\right) \geq \chi_{i}$ (for all $t$ ). Conversely, we have that $d\left(D_{t}, e^{*}\right)<\chi_{i}$ implies $d\left(A_{t}, e^{*}\right)<a$. Similarly, for edge $e$, we get that $d\left(D_{t}, e\right)<\chi_{i}$ implies $d\left(A_{t}, e\right)<a$. By the definition of $D_{t}$, we have that $d\left(D_{t}, e\right)<$ $\chi_{i}$ for $t \leq 0$, thus $d\left(A_{t}, e\right)<a$ for $t \leq 0$. Furthermore, the angle between $e$ and segment $\left\{A_{t} \mid t \in\left[-\chi^{-}, \chi^{+}\right]\right\}$is at least $\pi / 3$, because this angle is at least the angle between their orthogonal projection on the plane defined by $e$ and $D$ that is the angle between $e$ and $D$, since all $A_{t}$ lie in the plane spanned by $D_{t}$ which projects on $D$; the lower bound of $\pi / 3$ follows since the angle between $e$ and $D$ is at least $\pi / 3$ by the definition of $\Omega$. Hence, the locus of points $A_{t}$ for $t \leq 0$ is a segment that (i) intersects $e^{\prime}$ (since $D_{0}$ intersects $e^{\prime}$ ), (ii) lies in the half-plane containing $T_{i}$ and bounded by the line supporting $e$ (since $t \leq 0$ ), (iii) lies entirely within distance $a$ of $e$, and (iv) makes an angle of at least $\pi / 3$ with $e$; the locus of points $A_{t}$ for $t \leq 0$ thus lies in a region, denoted $\Upsilon$, shown in dark gray in Fig. 7(a). For proving that $d\left(A_{t}, e^{*}\right) \geq a$ for $t \leq 0$, it is thus sufficient to prove that this region does not intersect the set, denoted $\Upsilon^{\prime}$, of points at distance less than $a$ from $e^{*}$ (shown in light gray in Fig. 7(a)).

Referring to Figs. 7(b)-(c), let $p$ be the endpoint of $e^{\prime}$ closest to $e^{*}$ and $s$ be its projection on the line supporting $e^{*}$. If the two regions $\Upsilon$ and $\Upsilon^{\prime}$ intersect, there exists a point $q$ in the intersection that is at distance less than or equal to $\frac{2}{\sqrt{3}} a$ from $p$ and at distance less than or equal to $a$ from $e^{*}$; thus $d(p, s) \leq d\left(p, e^{*}\right) \leq d(p, q)+$ $d\left(q, e^{*}\right) \leq\left(1+\frac{2}{\sqrt{3}}\right) a$. On the other hand, $d(p, s)$ is one fourth of one of the heights of the triangle $T_{i}$ and thus is at least $\frac{h_{i}}{4}$. Hence, if the two regions intersect, then $\frac{h_{i}}{4} \leq\left(1+\frac{2}{\sqrt{3}}\right) \frac{\chi_{i}}{\sin \Gamma}$. We postpone to Lemma 7 the proof that, with $C=31.3 \beta$, we have $\frac{h_{i}}{4}>\left(1+\frac{2}{\sqrt{3}}\right) \frac{\chi_{i}}{\sin \Gamma}$, which implies that the two regions $\Upsilon$ and $\Upsilon^{\prime}$ are disjoint. This concludes the proof that no line $D_{t}$ intersects the boundary of $f_{n}\left(T_{e}\right)$.

We now prove that $D^{-}$intersects $f_{n}\left(T_{e}\right)$. Consider a projection, $p()$, along the direction $d$ onto a plane orthogonal to $d$. We proved that, for any of the two triangles 


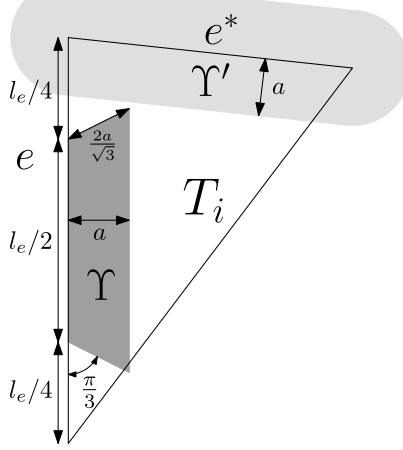

(a)

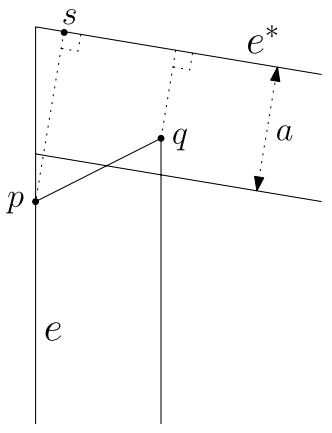

(b)

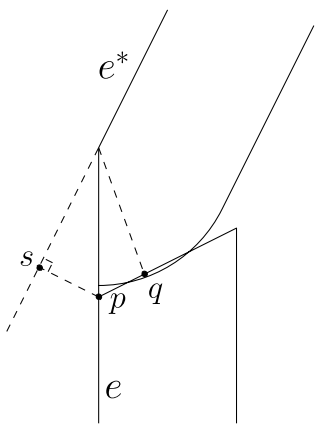

(c)

Fig. 7 For the proof of Lemma 4

Fig. 8 Projection of $\partial T_{i}$, $f_{n}\left(\partial T_{i}\right)$, and $D^{-}$

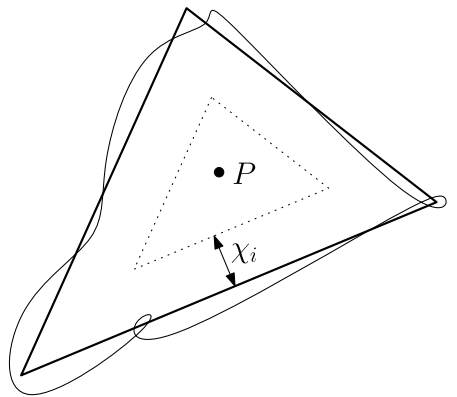

$T_{i}, \Upsilon$ is at distance at least $a=\frac{\chi_{i}}{\sin \Gamma} \geq \chi_{i}$ from each edge $e^{*} \neq e$ of $T_{i}$. It follows that $\Upsilon$ lies in triangle $T_{i}$ and thus that $D_{t}$ intersects $T_{i}$ for all $t \leq 0$. Therefore, $D^{-}$ intersects $T_{i}$ and is at distance at least $\chi_{i}$ from each edge $e^{*} \neq e$ of $T_{i}$ for $i=1,2$. Furthermore, $D^{-}$is at distance $\chi^{-}=\min \left(\chi_{1}, \chi_{2}\right)$ from $e$, by definition. We now consider the triangle $T_{i}$ for which $\chi_{i}=\chi^{-}$. It follows that $D^{-}$is at distance at least $\chi_{i}$ from all three edges of $T_{i}$. Thus $D^{-}$projects to a point $P=p\left(D^{-}\right)$inside triangle $p\left(T_{i}\right)$, at distance at least $\chi_{i}$ from the three edges of $p\left(T_{i}\right)$ (see Fig. 8), since distances to $D^{-}$are preserved by an orthogonal projection along its direction.

Roughly speaking, by Hypothesis 4 , the curve $f_{n}\left(\partial T_{i}\right)$ is at distance less than $\chi_{i}$ from $\partial T_{i}$ (the boundary of $T_{i}$ ), thus its projection $p\left(f_{n}\left(\partial T_{i}\right)\right)$ is at distance less than $\chi_{i}$ from the edges of $p\left(T_{i}\right)$. It is thus intuitively clear that $p\left(D^{-}\right)$intersects $p\left(f_{n}\left(T_{i}\right)\right)$ and thus that $D^{-}$intersects $f_{n}\left(T_{i}\right)$ (and thus $f_{n}\left(T_{e}\right)$ ).

More formally, consider the map $g_{n}$ from the triangle $p\left(T_{i}\right)$ to the plane containing it such that, for any point $x$ in $T_{i}$, the point $p(x)$ is sent to the point $g_{n}(p(x))=$ $p\left(f_{n}(x)\right)$. We first prove that the curves $p\left(\partial T_{i}\right)$ and $g_{n}\left(p\left(\partial T_{i}\right)\right)$ are homotopic in $\mathbb{R}^{2} \backslash P$. Consider the continuous map

$$
\begin{aligned}
F: & \partial T_{i} \times[0,1] \longrightarrow \mathbb{R}^{2} \\
& (x, \lambda) \longrightarrow \lambda p(x)+(1-\lambda) g_{n}(p(x))=\lambda p(x)+(1-\lambda) p\left(f_{n}(x)\right) .
\end{aligned}
$$


$F$ is a homotopy between the curves $p\left(\partial T_{i}\right)$ and $g_{n}\left(p\left(\partial T_{i}\right)\right)$ in $\mathbb{R}^{2}$. We prove that the image of $F$ does not contain $P$, which yields the result. The triangle inequality gives

$$
d(P, F(x, \lambda)) \geq d(P, p(x))-d(F(x, \lambda), p(x)) .
$$

We have already proved that the point $P$ is at distance at least $\chi_{i}$ from $p(x)$ for all points $x$ in $\partial T_{i}$. On the other hand, the distance between $p(x)$ and $p\left(f_{n}(x)\right)$ is larger than or equal to the distance between $p(x)$ and their barycenter $F(x, \lambda)$ for any $\lambda \in[0,1]$. Hence

$$
d(P, F(x, \lambda)) \geq \chi_{i}-d\left(p(x), p\left(f_{n}(x)\right)\right) .
$$

Finally, since $d\left(p(x), p\left(f_{n}(x)\right)\right)<\chi_{i}$ for all $x \in T_{i}$, by Hypothesis 4 , we have that $d(P, F(x, \lambda))>0$ for all $(x, \lambda)$. Hence, the image of $F$ does not contain point $P$, and thus the curves $p\left(\partial T_{i}\right)$ and $g_{n}\left(p\left(\partial T_{i}\right)\right)$ are homotopic in $\mathbb{R}^{2} \backslash P$.

Now, we can contract $p\left(\partial T_{i}\right)$ to a point while remaining in $p\left(T_{i}\right)$. Composing this with $g_{n}$ gives a contraction of $g_{n}\left(p\left(\partial T_{i}\right)\right)$ in $g_{n}\left(p\left(T_{i}\right)\right)$. On the other hand, there is no contraction of $p\left(\partial T_{i}\right)$ in $\mathbb{R}^{2} \backslash P$ (since $P$ is in $p\left(T_{i}\right)$ ), thus there is no contraction of its homotopic curve $g_{n}\left(p\left(\partial T_{i}\right)\right)$ in $\mathbb{R}^{2} \backslash P$. Hence, there exists a curve that is contractible in $g_{n}\left(p\left(T_{i}\right)\right)$ but not in $\mathbb{R}^{2} \backslash P$. It follows that $g_{n}\left(p\left(T_{i}\right)\right)$ is not included in $\mathbb{R}^{2} \backslash P$. Hence $P$ is in $g_{n}\left(p\left(T_{i}\right)\right)=p\left(f_{n}\left(T_{i}\right)\right)$. Therefore, $D^{-}$intersects $f_{n}\left(T_{i}\right)$ and thus $f_{n}\left(T_{e}\right)$, which concludes the proof.

We finally prove the two following simple technical lemmas, which complete the proof of Theorem 1 . Recall that $\Gamma$ is the smallest angle a direction $d \in \Omega$ can make with the plane containing $T_{i}$.

Lemma $6 \sin \Gamma=\frac{\sqrt{3}}{2} \sin \frac{C}{2 \sqrt{n}}$.

Proof In the following, we identify the sphere of directions with a sphere $\mathcal{S}$ embedded in $\mathbb{R}^{3}$; let $O$ denote its center. We assume that the embedding preserves directions (i.e., for any direction $d$, the corresponding point $M$ on $\mathcal{S}$ is such that $d$ and $O M$ have the same direction).

Refer to Fig. 9. Let $d$ be a direction in $\Omega$ and $M$ be its corresponding point on $\mathcal{S}$. Consider one of the $T_{i}$, and let $\mathcal{P}$ be the plane containing $O$ and parallel to the plane containing $T_{i}$. Let $H$ be the orthogonal projection of $M$ onto plane $\mathcal{P}$. Let $E$ and $E^{\prime}$ be the two points on $\mathcal{S}$ that correspond to the two (opposite) directions of segment $e$. Let $K$ be the orthogonal projection of $M$ (and $H$ ) onto the line $E E^{\prime}$. Finally, let $\theta$ be the angle $\angle M K H, \phi$ be the angle $\angle M O K$, and $\gamma$ be the angle $\angle M O H$.

It follows from these definitions that

$$
\sin \gamma=\frac{H M}{O M}=\frac{H M}{K M} \frac{K M}{O M}=\sin \theta \sin \phi .
$$

Now, the angle $\gamma$ is also the angle between direction $d$ and the plane that contains $T_{i}$. Thus $\Gamma=\inf _{d \in \Omega} \gamma$, by the definition of $\Gamma$. The angle $\theta$ is the angle between the plane containing $T_{i}$ and the plane containing $e$ and $d$. It thus follows from the definition of $\Omega$ that $\inf _{d \in \Omega} \theta=\frac{C}{2 \sqrt{n}}$ (see Fig. 5(a)). The angle $\phi$ is the angle between $d$ and 
Fig. 9 For the proof of Lemma 6

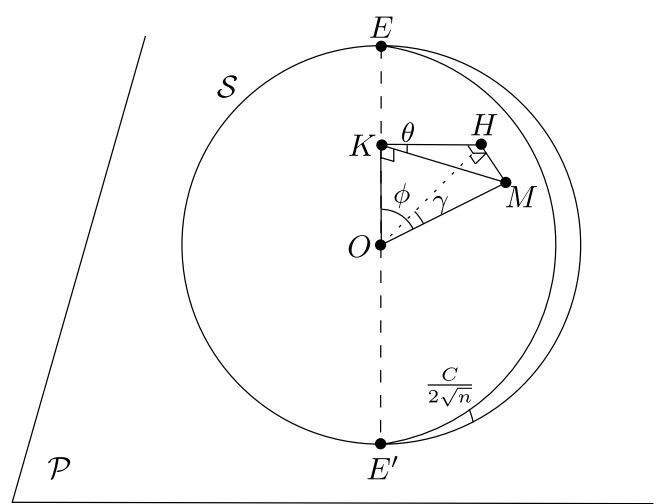

the line containing $e$. It thus also follows from the definition of $\Omega$ that $\inf _{d \in \Omega} \phi=\frac{\pi}{3}$. In addition, since $\gamma, \theta$, and $\phi$ are in $\left[0, \frac{\pi}{2}\right]$, we have

$$
\sin \Gamma=\inf _{d \in \Omega} \sin \gamma, \quad \inf _{d \in \Omega} \sin \theta=\sin \frac{C}{2 \sqrt{n}}, \quad \text { and } \quad \inf _{d \in \Omega} \sin \phi=\sin \frac{\pi}{3} .
$$

Furthermore, the constraints on $\theta$ and $\phi$ in the definition of $\Omega$ are independent. Thus, the minima of $\theta$ and $\phi$ can be attained for the same direction $d$ in $\Omega$. It follows that

$$
\inf _{d \in \Omega}(\sin \theta \sin \phi)=\inf _{d \in \Omega} \sin \theta \cdot \inf _{d \in \Omega} \sin \phi .
$$

We can thus conclude that

$$
\sin \Gamma=\inf _{d \in \Omega} \sin \gamma=\inf _{d \in \Omega} \sin \theta \sin \phi=\inf _{d \in \Omega} \sin \theta \inf _{d \in \Omega} \sin \phi=\frac{\sqrt{3}}{2} \sin \frac{C}{2 \sqrt{n}} .
$$

Lemma $7 \frac{h_{i}}{4}>\left(1+\frac{2}{\sqrt{3}}\right) \frac{\chi_{i}}{\sin \Gamma}$ with $C=31.3 \beta$.

Proof By Lemma 6, replacing $\chi_{i}$ and $\Gamma$ by their values in the inequality $\frac{h_{i}}{4}>$ $\left(1+\frac{2}{\sqrt{3}}\right) \frac{\chi_{i}}{\sin \Gamma}$ gives

$$
\frac{h_{i}}{4}>\left(1+\frac{2}{\sqrt{3}}\right) \frac{\frac{\beta h_{i}}{\sqrt{n}}}{\frac{\sqrt{3}}{2} \sin \left(\frac{C}{2 \sqrt{n}}\right)}
$$

or equivalently

$$
4 \beta\left(1+\frac{2}{\sqrt{3}}\right)<\sqrt{n} \frac{\sqrt{3}}{2} \sin \left(\frac{C}{2 \sqrt{n}}\right) .
$$

Notice first that for large enough values of $n$, using the approximation $\sin x \approx x$ in the neighborhood of zero, we derive the sufficient condition

$$
C>\frac{16 \beta}{\sqrt{3}}\left(1+\frac{2}{\sqrt{3}}\right) \sim 19.9 \beta .
$$


Now, since we want our result for all $n$, the computation is more complicated. Recall first that, for any strictly concave function $f$ such that $f(0)=0, f(x)>$ $\frac{f\left(x_{0}\right)}{x_{0}} x$ for any $x \in\left(0, x_{0}\right)$. It follows that $\sin x>\frac{2}{\pi} x$ for any $x \in\left(0, \frac{\pi}{2}\right)$. Since we assumed that $\theta_{e}-\frac{C}{\sqrt{n}}>0$ and thus that $0<\frac{C}{2 \sqrt{n}}<\frac{\theta_{e}}{2}<\frac{\pi}{2}$, we get

$$
\sin \left(\frac{C}{2 \sqrt{n}}\right)>\frac{2}{\pi} \frac{C}{2 \sqrt{n}} .
$$

To guarantee inequality (2), it is thus sufficient to have

$$
4 \beta\left(1+\frac{2}{\sqrt{3}}\right) \leq \sqrt{n} \frac{\sqrt{3}}{2} \frac{2}{\pi} \frac{C}{2 \sqrt{n}}
$$

or equivalently

$$
C \geq \frac{8}{3}(2+\sqrt{3}) \pi \beta \sim 31.27 \beta,
$$

which concludes the proof. Note that we can set

$$
C=31.3 \beta
$$

in the definition of $\Omega$ (in the proof of Lemma 3), since Inequality (2) is the only constraint on $C$.

\subsection{Generalizations}

We prove here Theorem 2. We first show that Theorem 1 generalizes to the case where the viewpoint is chosen randomly at finite distance. We then show that considering surfaces with boundary does not change the asymptotic expected complexity of the silhouette.

\subsubsection{Points of View at Finite Distance}

We have thus far restricted ourselves to the case where the viewpoint is chosen uniformly at random at infinity. However, still under Hypotheses 1-3 or 1 and 4, our result applies to any distribution of viewpoints such that the probability for an edge $e$ of $P_{n}$ to be on the transparent silhouette is bounded from above by $c \theta_{e}$ for some positive constant $c$, where $\theta_{e}$ is the exterior dihedral angle associated to $e$. Indeed, the expected number of edges on the silhouette is then at most $c \sum_{\text {edge } e} \theta_{e}$, and we get the result by applying, as before, Lemma $3 .^{2}$

Such a distribution of viewpoints is obtained, in particular, under Hypotheses 1-3 when the point of view is chosen uniformly at random in any given bounded region, $B$, of positive volume. Indeed, there exists, by Hypothesis 3 , a constant $R$ such that $B$ is included in any ball of radius $R$ centered at any point of polyhedron $P_{n}$. Furthermore, the probability that an edge $e$, with associated dihedral angle $\theta_{e}$, appears on the

\footnotetext{
${ }^{2}$ In Lemma $3, \operatorname{silh}\left(f_{n}\left(T_{e}\right)\right)$ always refers to an expected length for a viewpoint chosen randomly at infinity.
} 
silhouette viewed from a random point in $B$ is the ratio of the volume of the intersection of $B$ with a spherical double wedge of angle $\theta_{e}$ and radius $R$ (similarly as in Fig. 4(b)) over the volume of $B$ (which is a constant). The volume of this intersection is less than the volume of the spherical double wedge, which is $\frac{4}{3} \theta_{e} R^{3}$. Hence, the probability that edge $e$ appears on the silhouette is less than $c \theta_{e}$ for some constant $c$. The expected number of edges on the silhouette is thus at most $c \sum_{\text {edge } e} \theta_{e}$, which is, by Lemma 3, in $O(\sqrt{n})$ or, more precisely, at $\operatorname{most}^{3} c \pi\left(15 \beta+\frac{24}{\alpha} \operatorname{silh}(S)\right) \sqrt{n}$.

\subsubsection{Surfaces with Boundary}

Let $S$ be a 2-manifold with boundary $\mathcal{B}$. We consider that the boundary is always on the transparent silhouette, and so the definition of the transparent silhouette of a 2-manifold $S$ with boundary is exactly that of a 2-manifold without boundary plus the boundary $\mathcal{B}$.

The surface $S$ is approximated by a triangulated mesh $P_{n}$ that satisfies Hypotheses 1 and 4, as in the case without boundary, except that now the mesh may not be a polyhedron (some edges may have only one adjacent face rather than two).

To give an upper bound on the number of edges on the silhouette of the mesh, we consider the boundary edges and the other (nonboundary) edges separately. For the nonboundary edges, the same reasoning as before still holds. For the boundary edges, it is easy to see that the length (in 3D) of the boundary of $P_{n}$ cannot be much larger than the length of $\mathcal{B}$. Indeed, the two are homeomorphic, and Hypothesis 4 implies that the image by $f_{n}$ of an edge $e$, of length $l_{e}$, is a curve whose endpoints lie at distance at least $l_{e}-2 \frac{\beta l_{e}}{\sqrt{n}}$ apart. Hence, for $n$ sufficiently large, the length of $f_{n}(e)$ is at least $l_{e} / 2$, that is, at least $\frac{\alpha}{2 \sqrt{n}}$ by Hypothesis 1 . This means that the length of $\mathcal{B}$ is at least $\frac{\alpha}{2 \sqrt{n}}$ times the number of boundary edges of $P_{n}$. Hence, the number of boundary edges of $P_{n}$ is at most $\frac{2 \sqrt{n}}{\alpha}$ times the length of $\mathcal{B}$. So, if the length of $\mathcal{B}$ is bounded, the expected complexity of the silhouette of $P_{n}$ is $O(\sqrt{n})$.

\section{Conclusion}

This paper gives an idea of why, and when, the usual claim that the silhouette of a triangulated mesh has size $O(\sqrt{n})$ is valid. In particular, we gave a set of conditions such that any triangulated mesh of size $n$ approximating a surface in a way that satisfies those conditions has a silhouette of expected size $O(\sqrt{n})$. Roughly speaking, the mesh should have no short edges, its faces should be fat, and the distance between the mesh and the surface it approximates should never be too large. The surface itself is not necessarily everywhere differentiable and may have boundaries.

\footnotetext{
${ }^{3}$ Note that the constant $c$ can be very large. For instance, if $S$ is the surface of a conical frustum and $B$ is a small ball centered at the apex of the cone, we can find a sequence of $P_{n}$ that satisfy the hypotheses and have a silhouette of linear complexity viewed from the center of $B$ (as in Fig. 1). For $B$ small enough, the silhouette can be linear from any point in $B$ for all $n$ up to an arbitrarily large value. It is then natural that for a small $B$, the upper bound $c \pi\left(15 \beta+\frac{24}{\alpha} \operatorname{silh}(S)\right) \sqrt{n}$ on the average size of the silhouette is only smaller than $n$ for very large values of $n$.
} 
A natural question to ask is what kind of meshes satisfy those conditions. One such example, for smooth surfaces, is the meshes produced by Boissonnat and Oudot [2]. The critical property of the meshes they compute is that the ratio between the size of the largest and the smallest triangles remains bounded, although meshes are nonuniform with smaller triangles in areas of large curvature.

Many nonsmooth surfaces can also be approximated by meshes that satisfy our hypotheses. However, our conditions may force the mesh to contain many more triangles than needed to approximate the surface with a given precision. This is, in particular, the case for cylinders. Indeed, an economic way to approximate a (finite section of a) cylinder is with $n$ long and thin triangles. However, the triangles are not fat, and the short edges have length $\Theta\left(\frac{1}{n}\right)$. This contradicts our hypotheses (1 and 2), and, indeed, the silhouette has linear average complexity. To satisfy our hypotheses without making the precision of the approximation worse, we need to subdivide the long edges until they have roughly the same size as the short ones (see Fig. 1). This does not modify the shape of the silhouettes, but long edges on a silhouette are replaced by $\Theta(n)$ short edges. The expected complexity of silhouettes remains linear in $n$, but the total number of triangles becomes quadratic.

In computer graphics, meshes are often nonuniform and do not necessarily satisfy our hypotheses. This explains why the observed expected complexity of silhouettes may, as in [10], be larger than $O(\sqrt{n})$. Nonetheless, our result gives a good idea of why meshes, even when they are not perfectly uniform, appear to have silhouettes of sub-linear complexity.

An interesting question is whether our result on silhouettes extends to related structures such as suggestive contours [3] or apparent ridges [8], which were designed to convey more information about the visual shape of an object than the sole silhouette. It appears that our result does not extend to suggestive contours, because it relies on the fact that edges that lie in almost flat regions appear very rarely on the silhouette, whereas an almost flat region with arbitrarily small bumps everywhere has a suggestive contour of linear size from a set of viewpoints of measure $\Omega(1)$. The question remains, however, open for other structures such as apparent ridges.

Acknowledgements The authors wish to thank Gert Vegter, who introduced the problem to us, and gratefully acknowledge fruitful discussions on this topic with Helmut Alt, Olivier Devillers, Hazel Everett, Xavier Goaoc, Bruno Levy, and Sylvain Petitjean.

\section{References}

1. Alt, H., Glisse, M., Goaoc, X.: On the worst-case complexity of the silhouette of a polytope. In: 15 th Canadian Conference on Computational Geometry-CCCG'03, pp. 51-55 (2003)

2. Boissonnat, J.-D., Oudot, S.: Provably good sampling and meshing of surfaces. Graph. Models Special Issue Solid Model. 67(5), 405-451 (2005)

3. DeCarlo, D., Finkelstein, A., Rusinkiewicz, S., Santella, A.: Suggestive contours for conveying shape. ACM Trans. Graph. 22(3), 848-855 (2003)

4. Duguet, F.: Shadow computations using robust epsilon visibility. Research Report 5167, INRIA (2004)

5. Duguet, F., Drettakis, G.: Robust epsilon visibility. In: Hughes, J. (ed.) Proceedings of ACM SIGGRAPH 2002, pp. 567-575. ACM Press/ACM SIGGRAPH, New York (2002) 
6. Efrat, A., Guibas, L.J., Hall-Holt, O.A., Zhang, L.: On incremental rendering of silhouette maps of polyhedral scene. In: SODA '00: Proceedings of the Eleventh Annual ACM-SIAM Symposium on Discrete Algorithms, pp. 910-917. Society for Industrial and Applied Mathematics, Philadelphia (2000)

7. Isenberg, T., Freudenberg, B., Halper, N., Schlechtweg, S., Strothotte, T.: A developer's guide to silhouette algorithms for polygonal models. IEEE Comput. Graph. Appl. 23(4), 28-37 (2003)

8. Judd, T., Durand, F., Adelson, E.H.: Apparent ridges for line drawing. ACM Trans. Graph. 26(3), 19 (2007)

9. Kettner, L., Welzl, E.: Contour edge analysis for polyhedron projections. In: Geometric Modeling: Theory and Practice, pp. 379-394. Springer, Berlin (1997)

10. McGuire, M.: Observations on silhouette sizes. J. Graph. Tools 9(1), 1-12 (2004)

11. Sander, P.V., Gu, X., Gortler, S.J., Hoppe, H., Snyder, J.: Silhouette clipping. In: SIGGRAPH '00: Proceedings of the 27th annual conference on Computer graphics and interactive techniques, pp. 327334. ACM Press/Addison-Wesley, New York (2000)

12. Schwarz, H.A.: Sur une définition erronée de l'aire d'une surface courbe. In: Gesammelte Mathematische Abhandlungen, vol. 1, pp. 309-311. Springer, Berlin (1890) 